\title{
Characterization of a CLYC Detector and Validation of the Monte Carlo Simulation by Measurement Experiments
}

\author{
Hyun Suk Kim ${ }^{1,2}$, Martin B. Smith ${ }^{3}$, Martin R. Koslowsky ${ }^{3}$, Sung-Woo Kwak', Sung-Joon Ye ${ }^{1,2,5}$, Geehyun Kim ${ }^{6, *}$ \\ ${ }^{1}$ Program in Biomedical Radiation Sciences, Department of Transdisciplinary Studies, Graduate School of Convergence Science and Technology, Seoul \\ National University, Seoul, Korea; ${ }^{2}$ Seoul National University Hospital, Seoul, Korea; ${ }^{3}$ Bubble Technology Industries Inc., Chalk River, ON, Canada; ${ }^{4}$ Policy \\ and Technology Research Division, Korea Institute of Nuclear Nonproliferation And Control (KINAC), Daejeon, Korea; ${ }^{5}$ Advanced Institutes of Convergence \\ Technology, Seoul National University, Suwon, Korea; ${ }^{6}$ Department of Nuclear Engineering, Sejong University, Seoul, Korea
}

\section{Original Research}

Received October 14, 2016

Revision December 9, 2016

Accepted December 29, 2016

Corresponding author: Geehyun Kim

Department of Nuclear Engineering, Sejong University, 209 Neungdong-ro, Gwangjin-gu, Seoul 05006, Korea

Tel: +82-2-3408-4013

Fax: +82-2-3408-3182

E-mail:gkim01@sejong.ac.kr

This is an Open-Access article distributed under the terms of the Creative Commons Attribution NonCommercial License (http://creativecommons.org/ licenses/by-nc/4.0) which permits unrestricted noncommercial use, distribution, and reproduction in any medium, provided the original work is properly cited.

Copyright $\odot$ 2017 The Korean Association for Radiation Protection

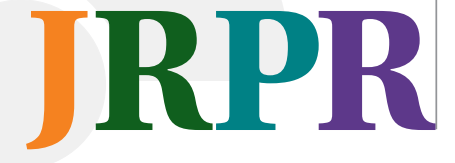

Background: Simultaneous detection of neutrons and gamma rays have become much more practicable, by taking advantage of good gamma-ray discrimination properties using pulse shape discrimination (PSD) technique. Recently, we introduced a commercial CLYC system in Korea, and performed an initial characterization and simulation studies for the CLYC detector system to provide references for the future implementation of the dual-mode scintillator system in various studies and applications.

Materials and Methods: We evaluated a CLYC detector with $95 \%{ }^{6} \mathrm{Li}$ enrichment using various gamma-ray sources and a ${ }^{252} \mathrm{Cf}$ neutron source, with validation of our Monte Carlo simulation results via measurement experiments. Absolute full-energy peak efficiency values were calculated for gamma-ray sources and neutron source using MCNP6 and compared with measurement experiments of the calibration sources. In addition, behavioral characteristics of neutrons were validated by comparing simulations and experiments on neutron moderation with various polyethylene $(\mathrm{PE})$ moderator thicknesses.

Results and Discussion: Both results showed good agreements in overall characteristics of the gamma and neutron detection efficiencies, with consistent $~ 20 \%$ discrepancy. Furthermore, moderation of neutrons emitted from ${ }^{252} \mathrm{Cf}$ showed similarities between the simulation and the experiment, in terms of their relative ratios depending on the thickness of the PE moderator.

Conclusion: A CLYC detector system was characterized for its energy resolution and detection efficiency, and Monte Carlo simulations on the detector system was validated experimentally. Validation of the simulation results in overall trend of the CLYC detector behavior will provide the fundamental basis and validity of follow-up Monte Carlo simulation studies for the development of our dual-particle imager using a rotational modulation collimator.

Keywords: CLYC, Pulse shape discrimination, Detection efficiency, Monte Carlo simulation, Dual-particle imager

\section{Introduction}

In the last few years, several new scintillator materials have been developed for the simultaneous detection of neutrons and gamma rays, taking advantage of good gammaray discrimination properties using pulse shape discrimination (PSD) technique [1-4]. Elpasolite scintillators present excellent performances in the identification of gamma rays and neutrons, and $\mathrm{CLYC}\left(\mathrm{Cs}_{2} \mathrm{LiYCl}_{6}: \mathrm{Ce}\right)$ is one of the elpasolite crystals, such as 
CLLC ( $\left.\mathrm{Cs}_{2} \mathrm{LiLaCl}_{6}: \mathrm{Ce}\right)$ and $\mathrm{CLLB}\left(\mathrm{Cs}_{2} \mathrm{LiLaBr}_{6}: \mathrm{Ce}\right)$. By virtue of its excellent ability to discriminate gamma rays from neutrons by PSD, along with good scintillation efficiencies for gamma rays, enormous interests and attentions have been paid to CLYC recently, in the field of radiation detection and measurement. According to the previous study, the light yield of CLYC has been reported to be around 20,000 photons/ $\mathrm{MeV}$, and it also exhibits an excellent proportionality between the deposited energy and the light output, which leads to good energy resolution for gamma rays ( $<5 \%$ at $662 \mathrm{keV}$ ).

Recently, we obtained a commercial CLYC system from Bubble Technology Industries (BTI) in Canada, and this is the first case of introducing a CLYC scintillator system in Korea. Therefore, we performed an initial characterization and simulation studies for the CLYC detector system to provide references for the future implementation of the dual-mode scintillator system in various studies and applications. In this paper, we report simulation and measurement experiment results of gamma rays and neutrons using the CLYC detector. The CLYC detector system from BTI was modeled and implemented into MCNP6 simulation to calculate the detection efficiency curves of gamma rays [5]. Neutron detection measurement experiments varying thicknesses of the polyethylene (PE) moderator was simulated for the ${ }^{252} \mathrm{Cf}$ source to find the optimal thickness of the PE moderator. Simulation results were validated by the measurement experiments performed at Korea Institute of Nuclear Nonproliferation and Control (KINAC).

In general, overall characteristics of the efficiency curves and the optimal moderator thicknesses showed similarity between the simulation and the measurement. However, there existed a certain amount of discrepancy due to the inevitable loss of counts caused by inherent nature of scintillators and pulse processing, implementation of PSD in measurement experiments, the limitation of the experimental environment in detail into the simulation, and the lack of precision in ${ }^{252} \mathrm{Cf}$ source modeling with considerations of alpha and gamma ray emission, as well as secondary processes in the simulation. Characterization results reported in this paper will provide the fundamental basis for future operation of the CLYC detector in the development of dual-mode radiation imager employing rotational modulation collimator (RMC) [6], in terms of the detection efficiency and the validity of follow-up Monte Carlo simulations for the modulation patterns.

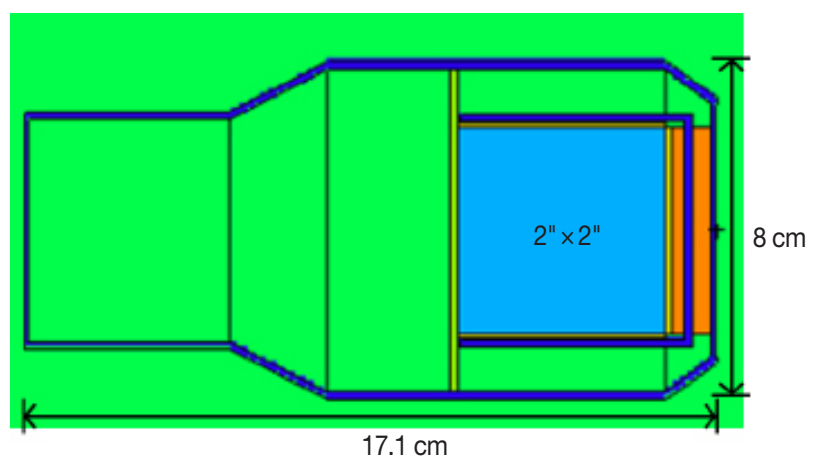

Fig. 1. A schematic of the CLYC detector system modeled in MCNP6.

\section{Materials and Methods}

\section{Modeling of the CLYC Detector System}

In this study, we performed Monte Carlo simulations using MCNP6 code system to calculate the detection efficiencies of the CLYC detector for gamma rays and neutrons. The geometry of the CLYC detector system supplied by BTI was encoded into an MCNP input, as a schematic shown in Figure 1 . The CLYC detector system consists of a 2 " $\times 2$ " cylindrical CLYC crystal, coupled to a R6233-100 photomultiplier tube by Hamamatsu (Shizuoka, Japan), signal processing electronics and firmware that perform PSD, and output connections, packaged altogether in a hand-held size device. The CLYC crystal is enriched to $95 \%$ in ${ }^{6} \mathrm{Li}$, wrapped by Teflon, and enclosed in an aluminum casing.

Precise modeling of the CLYC detector system is important, in regards of the subsequent development of the dualparticle imager based on the CLYC detector system [7]. Based on this work, we plan to perform Monte Carlo simulations on RMC to obtain simulated modulation patterns, depending on the source configuration, which will be used to reconstruct the image of the radioactive source distribution. In order to obtain physically meaningful modulation pattern results, we have to ensure precise modeling of the detector system, which has to be validated by experimental work. In particular, the modulation pattern consists of the number of incident radiations recorded in the detector depending on the rotational status of RMC, and the detection efficiency of the detection system plays an important role for the generation of the modulation patterns both in simulations and experiments. In addition, the physical nature of the incident radiations - neutron scattering and moderation, as well as gamma-ray interactions - embedded in the Monte Carlo simula- 
Table 1. A list of Gamma-ray Sources used in the Measurement Experiment

\begin{tabular}{lccc}
\hline Source & Activity $(\mathrm{Bq})$ & Reference date & Half-life (day) \\
\hline${ }^{133} \mathrm{Ba}$ & 393680 & $2016-04-01$ & 3,862 \\
${ }^{54} \mathrm{Mn}$ & 395160 & $2016-04-01$ & 312 \\
${ }^{137} \mathrm{Cs}$ & 378510 & $2016-04-01$ & 11,012 \\
${ }^{22} \mathrm{Na}$ & 358752 & $2016-04-01$ & 951 \\
${ }^{60} \mathrm{Co}$ & 380730 & $2016-04-01$ & 1,924 \\
\hline
\end{tabular}

tion must be validated, in order for us to utilize the simulation results for the fundamental basis in the design of the dual-particle RMC. In this paper, we calculated the gamma and neutron detection efficiencies of the CLYC detector system we employed for the development of the dual-particle imager based on the simulation geometry model, and the moderating characteristics of neutrons emitted from a ${ }^{252} \mathrm{Cf}$ source were simulated. The simulation results are to be validated by experiments, as will be discussed in the later sections.

\section{Simulation and Experiments on the Gamma-ray Detection Efficiency}

Based on the CLYC detector model aforementioned, gamma-ray detection efficiencies of the CLYC detector were simulated for various gamma-ray energies. We first assumed a point source of gamma rays located at a distance of $25 \mathrm{~cm}$ from the front face of the detector, and simulated $1 \times 10^{8}$ particle track histories for each gamma-ray source. In the simulation, we considered gamma rays emitted from ${ }^{137} \mathrm{Cs},{ }^{60} \mathrm{Co}$, ${ }^{133} \mathrm{Ba},{ }^{54} \mathrm{Mn}$, and ${ }^{22} \mathrm{Na}$ nuclides, and the pulse height tally (F8) in MCNP6 was used to distinguish full-energy absorption events of the gamma rays in the CLYC detector. Number of tallies obtained for each simulated gamma ray source were used to calculate the absolute full-energy absorption peak efficiency of the CLYC detector. Accordingly, one can also calculate the intrinsic peak efficiency of the detector by compensating the solid angle subtended by the 2"-diameter CLYC detector.

The simulation configuration was further refined by modeling gamma-ray sources which were actually used later in the measurement experiment; Table 1 provides the source information on the gamma-ray sources used in the experiment. The gamma-ray sources are standard calibration sources of R-type rod produced by Eckert \& Ziegler in Germany. Radioactive material is contained in a $4.75 \mathrm{~mm}$-diameter hole located in the cap area of a $127 \mathrm{~mm}$ high $\times 12.7 \mathrm{~mm}$ diameter plastic rod. Detailed geometry of the rod sources was also modeled and implemented in the Monte Carlo simulations.

Gamma-ray source measurement experiments were per-

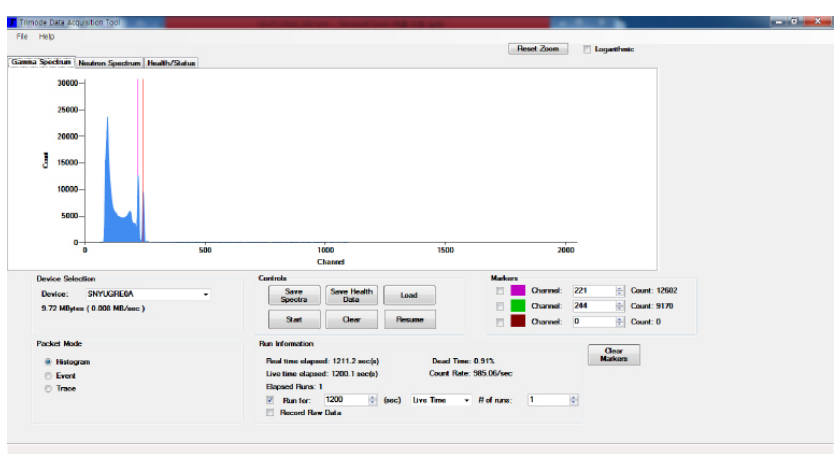

Fig. 2. A screenshot of the Trimode Data Acquisition Tool developed by BTI.
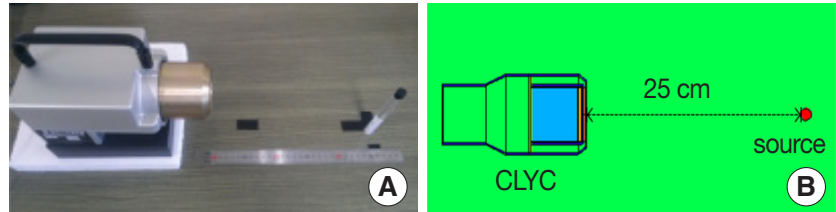

Fig. 3. (A) An experimental set-up for the gamma-ray source measurement and (B) a schematic of the MCNP6 simulation geometry.

formed using the Trimode v.1.0.16 data acquisition software provided by BTI, to collect gamma-ray and neutron signals with PSD. The appearance of the Trimode software is shown in Figure 2. We measured the background radiation for 6 hours, and measured ${ }^{137} \mathrm{Cs},{ }^{60} \mathrm{Co},{ }^{133} \mathrm{Ba},{ }^{54} \mathrm{Mn}$, and ${ }^{22} \mathrm{Na}$ sources listed in Table 1 for 20 minutes per source in live time. Sources were located at a distance of $25 \mathrm{~cm}$ from the front face of the detector, and the vertical location of the active area of radiation sources were aligned at the same height with the center of the CLYC detector. Figure 3 shows the measurement experiment set-up and a schematic of the MCNP6 simulation geometry. The dead time of the measurement was maintained under $1 \%$ for all measurements.

Gamma-ray spectra of each source were obtained and analyzed to calculate the peak area under the full-energy absorption peak. Absolute full-energy absorption peak efficiencies were calculated by the following equation [8]:

$$
\varepsilon(E)=\frac{A^{i}}{\Lambda^{i} \cdot Y(E) \cdot t} \cdot K_{i}
$$

where $A^{i}$ is the peak area of the radionuclide $\mathrm{i}, \Lambda^{i}$ is the reference activity of the radionuclide i, $Y(E)$ is the emission probability of the radionuclide at energy $\mathrm{E}, t$ is the spectrum collection time (live time) and $K_{i}$ is the decay correction factor which is defined using the difference between the reference date of the source and the experimental date. 


\section{Simulation and Experiments on the Neutron Moderation and the Detection Efficiency}

Simulations on behavioral characteristics of neutrons were also performed and verified by the ${ }^{252} \mathrm{Cf}$ neutron source measurement experiments. In the CLYC detector, a triton and an alpha particle are produced by the neutron capture reaction with ${ }^{6} \mathrm{Li}$; however, reaction libraries for alpha particle production in the neutron capture reaction were not available in the default MCNP data libraries. Therefore, the number of tritons created in CLYC was counted instead, using the F4/ FM tally, to calculate the neutron response by the CLYC scintillator [9]. For each neutron simulation hereafter, $1 \times 10^{8}$ particle track histories from the source were simulated.

First, we calculated the intrinsic detection efficiency of the CLYC detector system for an ideal thermal neutron source by MCNP6. A point source of $0.025 \mathrm{eV}$ thermal neutron located at $25 \mathrm{~cm}$ distance from the front face of the CLYC detector was assumed, and the number of thermal neutron capture reactions in the CLYC detector volume was tallied.

We then modeled the ${ }^{252} \mathrm{Cf}$ source used in the measurement experiment at KINAC. The ${ }^{252} \mathrm{Cf}$ source was an N-252 model (A3014-01 capsule type) produced by Eckert \& Ziegler in Germany. The source body was in a cylindrical shape with $36.32 \mathrm{~mm}$ in height and $9.4 \mathrm{~mm}$ in diameter, and the radioactive material was contained in the $1.57 \mathrm{~mm}$-diameter region at the center of the rod. The activity of ${ }^{252} \mathrm{Cf}$ source was 3.33 MBq on the reference date of 15 Oct. 2015. The energy spectrum of neutrons emitted from ${ }^{252} \mathrm{Cf}$ was assumed to follow the Watt fission spectrum [9-11].

Numbers of thermal neutrons measured by CLYC were calculated varying the thickness of the PE moderator, and measurement experiments were performed accordingly as shown in Figure 4A. We measured thermal neutrons from the ${ }^{252} \mathrm{Cf}$ source with 11 different thicknesses of the PE moderator. The dead time of the measurement was also under $1 \%$ for all measurements. For the simulation, environmental conditions of the experiment were attempted to be modeled as
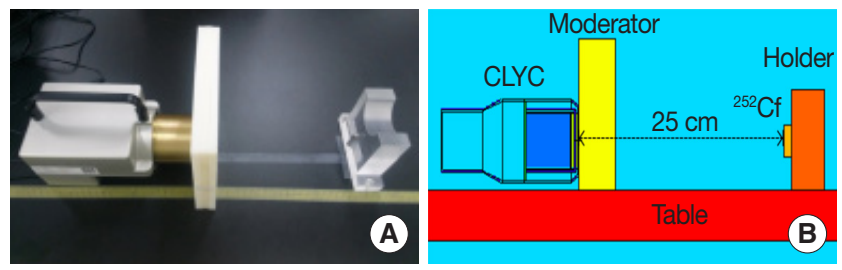

Fig. 4. (A) An experimental set-up for the neutron source measurement and (B) a schematic of the MCNP6 simulation geometry. close as possible to a reasonable content, which include, assuming the workbench table as a $5-\mathrm{cm}$ thick table made of polycarbonate, wood, or concrete, and modeling the polycarbonate source holder shown in the schematic illustrated in Figure 4B. Other environmental factors were not considered.

\section{Results and Discussion}

\section{Results on the Gamma-ray Detection Efficiency}

Gamma-ray energy spectra measured by CLYC detector are shown in Figure 5. Two sets of gamma-ray source measurement experiments were conducted on different days; however, spectral results from both measurements were identical. The energy resolution of full-energy absorption peaks was about $5 \%$ for most of gamma-ray sources we measured. In detail, the values were $8.3 \%, 5.6 \%, 5.3 \%, 4.4 \%, 4.0 \%$, and $3.8 \%$ for $356 \mathrm{keV}, 662 \mathrm{keV}, 835 \mathrm{keV}, 1,173 \mathrm{keV}, 1,275 \mathrm{keV}$, and $1,333 \mathrm{keV}$ peaks respectively.

We first validated our MCNP simulation results on the gamma-ray detection efficiency by comparing with detection efficiency simulation results calculated earlier at BTI. These earlier simulations used GEANT4 to calculate the absolute peak efficiency of a bare 2" $\times 2$ " CLYC crystal without any packaging, assuming a point source located $200 \mathrm{~cm}$ from the crystal face. Our CLYC detector model in MCNP6 simulation was able to generate results that are consistent with GEANT4 simulation of bare crystal. Figure 6 shows the comparison between two results, showing good agreement with each other. Slight differences are expected to be related to the internal structure of the CLYC detector model in MCNP6, as opposed to the bare CLYC crystal modeled in GEANT4.

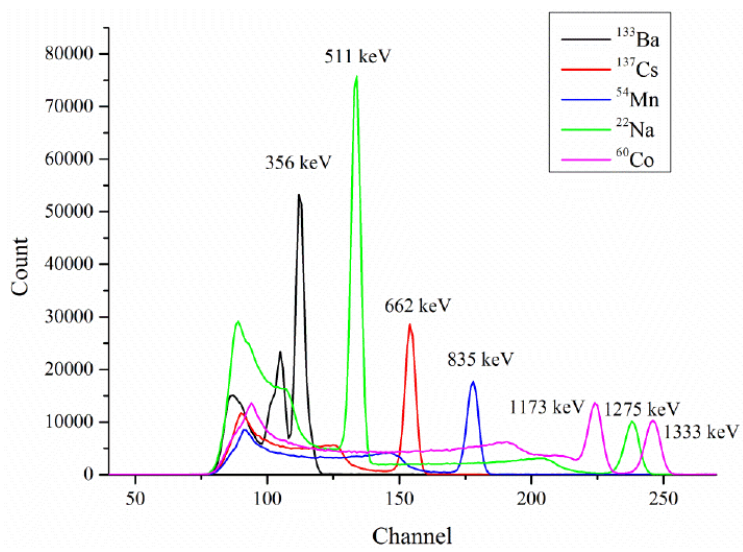

Fig. 5. Gamma-ray energy spectra measured by the CLYC detector system. 
Figure 7 shows (A) absolute and (B) intrinsic peak efficiencies for various gamma-ray sources simulated with MCNP6 and measured with the CLYC detector system modeled in the simulation. Detailed values of simulation and measurement results are given in Table 2. Error bars in Figure 7 indicate 95\% confidence intervals on the simulated and experimental results. A relative error of $3 \%$ in the gamma-ray source activity, which was indicated in the reference source certificate, was also considered in the error bars via error propagation.

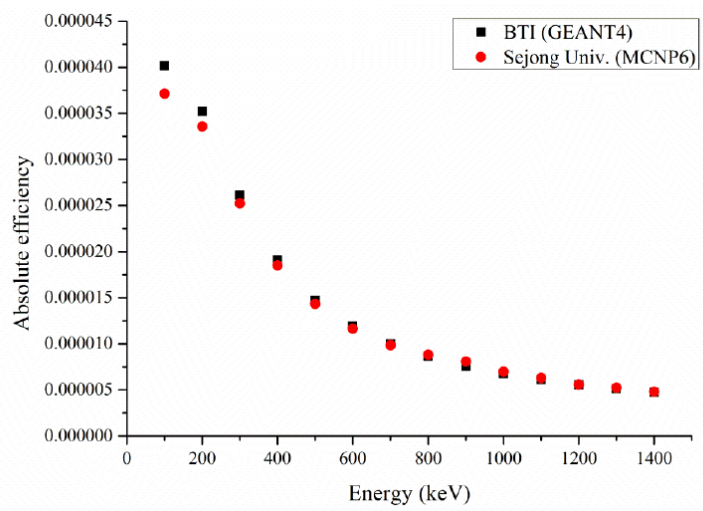

Fig. 6. Comparison of absolute efficiencies calculated by BTI (using GEANT4, shown in black squares) and Sejong University. (using MCNP6, shown in red circles).

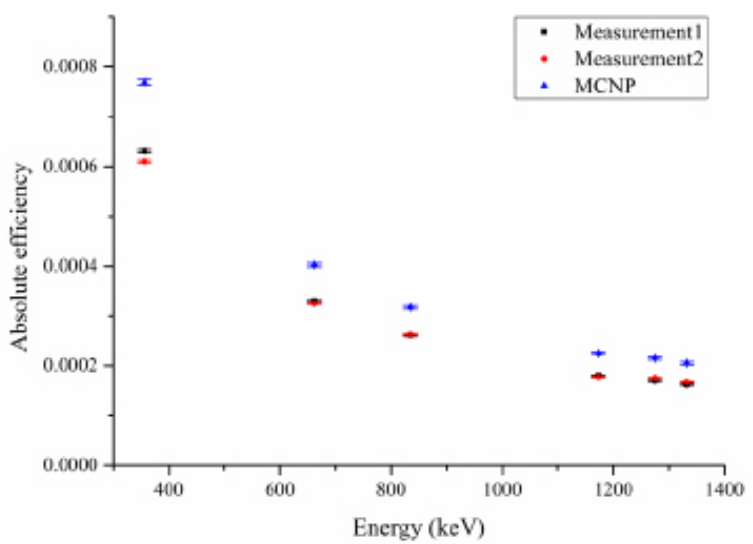

Measured peak efficiencies were generally lower than simulated results, showing $\sim 80 \%$ values of the simulation results for overall energy range. Simulated absolute efficiency values multiplied by 0.8 , are shown as "Modified MCNP" field in Table 2. Modified MCNP values show very good agreements with the first and the second measurement results.

The CLYC detector system from BTI utilizes a method that compares the leading edge of each pulse to the total integrated signal, in order to distinguish neutron signals from gammas. For low energy gammas, the pulse amplitude in general is not large enough for appropriate discriminations of the pulse shape, and discrimination of gammas and neutrons becomes difficult.

Figure 8 shows gamma-ray energy spectra of ${ }^{109} \mathrm{Cd}$ and ${ }^{57} \mathrm{Co}$ sources. In the case of ${ }^{109} \mathrm{Cd}$, we could not collect any counts from the $88.04 \mathrm{keV}$ peak (channel \# 76) because of the low-energy threshold in the CLYC detector system. When the background spectrum is subtracted from the measured spectrum, there weren't any counts left in the relevant channels that can give any statistical significance to discriminate the gamma peak. This was confirmed by two measurement experiments. In addition, in the case of ${ }^{57} \mathrm{Co}$, there were enough counts collected in the channels relevant to the 122

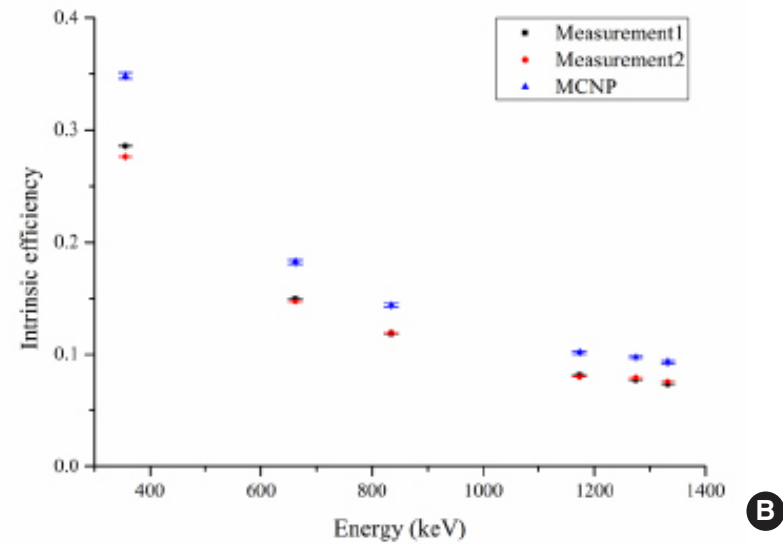

Fig. 7. Simulated and measured gamma-ray detection efficiencies of CLYC: (A) absolute efficiency and (B) intrinsic efficiency for various gamma-ray energy peaks.

Table 2. Simulation and Measurement Results on the Absolute Gamma-ray Detection Efficiency

\begin{tabular}{lcccc}
\hline Energy $(\mathrm{keV})$ & Measurement1 & Measurement2 & MCNP & Modified MCNP \\
\hline 356 & $6.31 \times 10^{-4}$ & $6.10 \times 10^{-4}$ & $7.70 \times 10^{-4}$ & $6.16 \times 10^{-4}$ \\
662 & $3.30 \times 10^{-4}$ & $3.25 \times 10^{-4}$ & $4.03 \times 10^{-4}$ & $3.22 \times 10^{-4}$ \\
835 & $2.61 \times 10^{-4}$ & $2.62 \times 10^{-4}$ & $3.17 \times 10^{-4}$ & $2.54 \times 10^{-4}$ \\
1173 & $1.80 \times 10^{-4}$ & $1.78 \times 10^{-4}$ & $2.25 \times 10^{-4}$ & $1.80 \times 10^{-4}$ \\
1275 & $1.69 \times 10^{-4}$ & $1.75 \times 10^{-4}$ & $2.15 \times 10^{-4}$ & $1.72 \times 10^{-4}$ \\
1333 & $1.61 \times 10^{-4}$ & $1.67 \times 10^{-4}$ & $2.05 \times 10^{-4}$ & $1.64 \times 10^{-4}$ \\
\hline
\end{tabular}



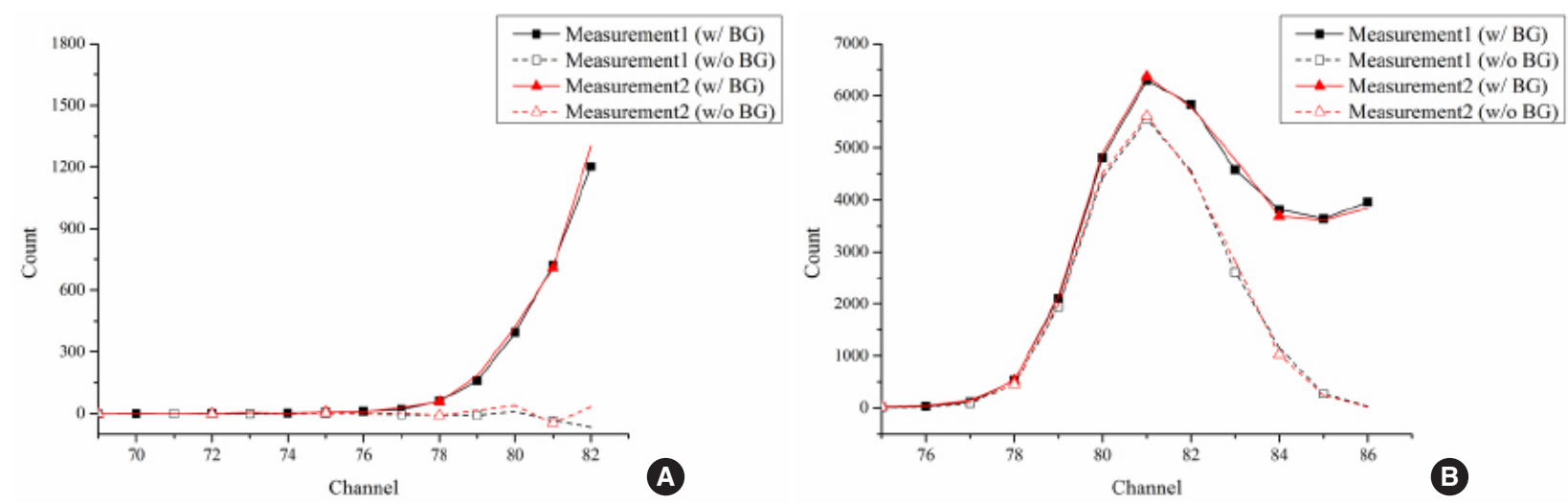

Fig. 8. Gamma-ray spectra of: (A) ${ }^{109} \mathrm{Cd}$ and $(B){ }^{57} \mathrm{Co}$, measured with the CLYC detector system.

$\mathrm{keV}$ peak (channel \# 81) to discriminate the feature; however, calculated detection efficiency based on the measured counts gave values far below what was expected from the MCNP simulation. The low-energy discrimination level of the detector system that is internally set to distinguish valid radiation signatures from noise could cut off pulses from low energy gamma rays. Ambiguity in pulse shape discrimination becomes more significant when the pulse amplitude is smaller. In that procedure, the system inevitably has to rule out some portion of valid full-energy absorption pulses.

However, starting from $356 \mathrm{keV}$ emitted from ${ }^{133} \mathrm{Ba}$ source, the simulation results exhibit consistent agreement with experimental results on detection efficiencies only with constant fraction difference, as shown in Figure 7. Therefore, we can make a reasonable estimation on the response of the real CLYC detector system based on simulation results.

\section{Results on the Neutron Moderation and the Detection Efficiency}

To verify our MCNP6 approach, we first compared the neutron detection efficiency value we calculated with those reported in previous literature. We obtained $76.2 \%$ intrinsic efficiency for bare 2" $\times 2$ " CLYC crystal without packaging, and the value was similar to a previous study. In the previous study, CLYC with the Li component enriched to $95 \%$ in ${ }^{6} \mathrm{Li}$ provided $\sim 80 \%$ intrinsic efficiency for thermal neutrons [4]. As we did not consider experimental environments in this calculation, contributions from the build-up effect could have been ignored, thus, the simulation result may underestimate the intrinsic detection efficiency. Other than this, two results make agreements with differences in a reasonable range; we concluded that this CLYC detector model will deliver reasonable estimations on the detector response to neutrons.

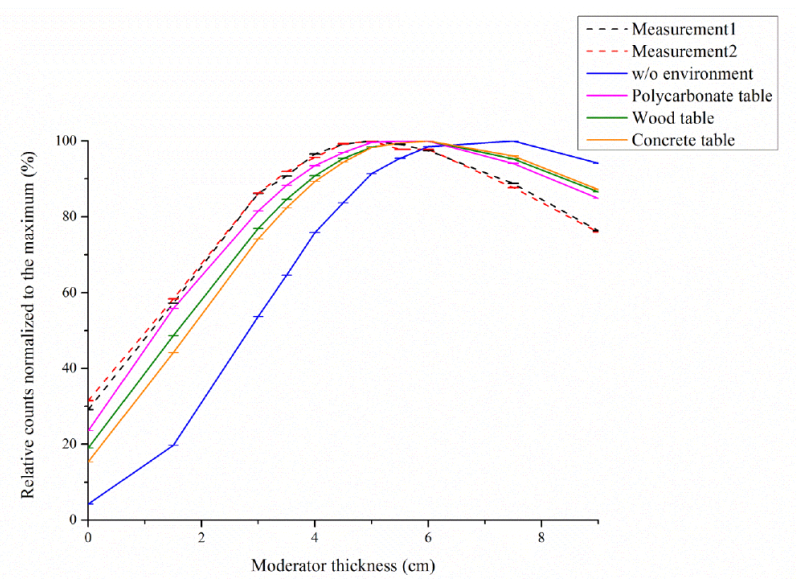

Fig. 9. Simulated and measured thermal neutron counts normalized to the maximum count in each set-up, obtained for various thicknesses of PE moderator; w/o environment means the table and the source holder were not considered in the simulation.

MCNP6 simulations and measurement experiments were performed to obtain neutron counts from ${ }^{252} \mathrm{Cf}$ source with PE moderators of various thicknesses. Two sets of measurement experiments were also performed on different days, to verify possible significant dependency of the system operation on the experimental environment; however, results from both measurements were almost the same. Figure 9 shows counts of thermal neutrons (after thermalization by the PE moderator) for various PE moderator thicknesses, normalized to the maximum count obtained by each measurement, and MCNP6 simulation. Without consideration of the experimental environment, simulated neutron counts changing over PE moderator thicknesses did not make good agreements with results from experiments, in terms of the overall trend of the curve. The optimal thickness of PE moderator that can give the maximum counts of thermalized 
Table 3. Simulation and Measurement Results on the Absolute Neutron Detection Efficiency

\begin{tabular}{lcccccc}
\hline Moderator thickness $(\mathrm{cm})$ & Measurement & Measurement2 & w/o environment & Polycarbonate & Wood & Concrete \\
\hline 0 & $1.88 \times 10^{-4}$ & $2.06 \times 10^{-4}$ & $1.17 \times 10^{-4}$ & $1.85 \times 10^{-4}$ & $1.26 \times 10^{-4}$ & $1.03 \times 10^{-4}$ \\
1.5 & $3.69 \times 10^{-4}$ & $3.81 \times 10^{-4}$ & $5.48 \times 10^{-4}$ & $4.39 \times 10^{-4}$ & $3.21 \times 10^{-4}$ & $2.97 \times 10^{-4}$ \\
3 & $5.55 \times 10^{-4}$ & $5.63 \times 10^{-4}$ & $1.49 \times 10^{-4}$ & $6.41 \times 10^{-4}$ & $5.08 \times 10^{-4}$ & $4.98 \times 10^{-4}$ \\
3.5 & $5.85 \times 10^{-4}$ & $6.00 \times 10^{-4}$ & $1.80 \times 10^{-4}$ & $6.94 \times 10^{-4}$ & $5.59 \times 10^{-4}$ & $5.54 \times 10^{-4}$ \\
4 & $6.23 \times 10^{-4}$ & $6.25 \times 10^{-4}$ & $2.11 \times 10^{-4}$ & $7.34 \times 10^{-4}$ & $6.00 \times 10^{-4}$ & $6.00 \times 10^{-4}$ \\
4.5 & $6.39 \times 10^{-4}$ & $6.49 \times 10^{-4}$ & $2.33 \times 10^{-4}$ & $7.62 \times 10^{-4}$ & $6.31 \times 10^{-4}$ & $6.34 \times 10^{-4}$ \\
5 & $6.45 \times 10^{-4}$ & $6.53 \times 10^{-4}$ & $2.54 \times 10^{-4}$ & $7.83 \times 10^{-4}$ & $6.50 \times 10^{-4}$ & $6.61 \times 10^{-4}$ \\
5.5 & $6.39 \times 10^{-4}$ & $6.39 \times 10^{-4}$ & $2.65 \times 10^{-4}$ & $7.86 \times 10^{-4}$ & $6.57 \times 10^{-4}$ & $6.68 \times 10^{-4}$ \\
6 & $6.28 \times 10^{-4}$ & $6.39 \times 10^{-4}$ & $2.74 \times 10^{-4}$ & $7.86 \times 10^{-4}$ & $6.61 \times 10^{-4}$ & $6.72 \times 10^{-4}$ \\
7.5 & $5.72 \times 10^{-4}$ & $5.73 \times 10^{-4}$ & $2.78 \times 10^{-4}$ & $7.39 \times 10^{-4}$ & $6.30 \times 10^{-4}$ & $6.46 \times 10^{-4}$ \\
9 & $4.92 \times 10^{-4}$ & $4.96 \times 10^{-4}$ & $2.62 \times 10^{-4}$ & $6.67 \times 10^{-4}$ & $5.72 \times 10^{-4}$ & $5.86 \times 10^{-4}$ \\
\hline
\end{tabular}
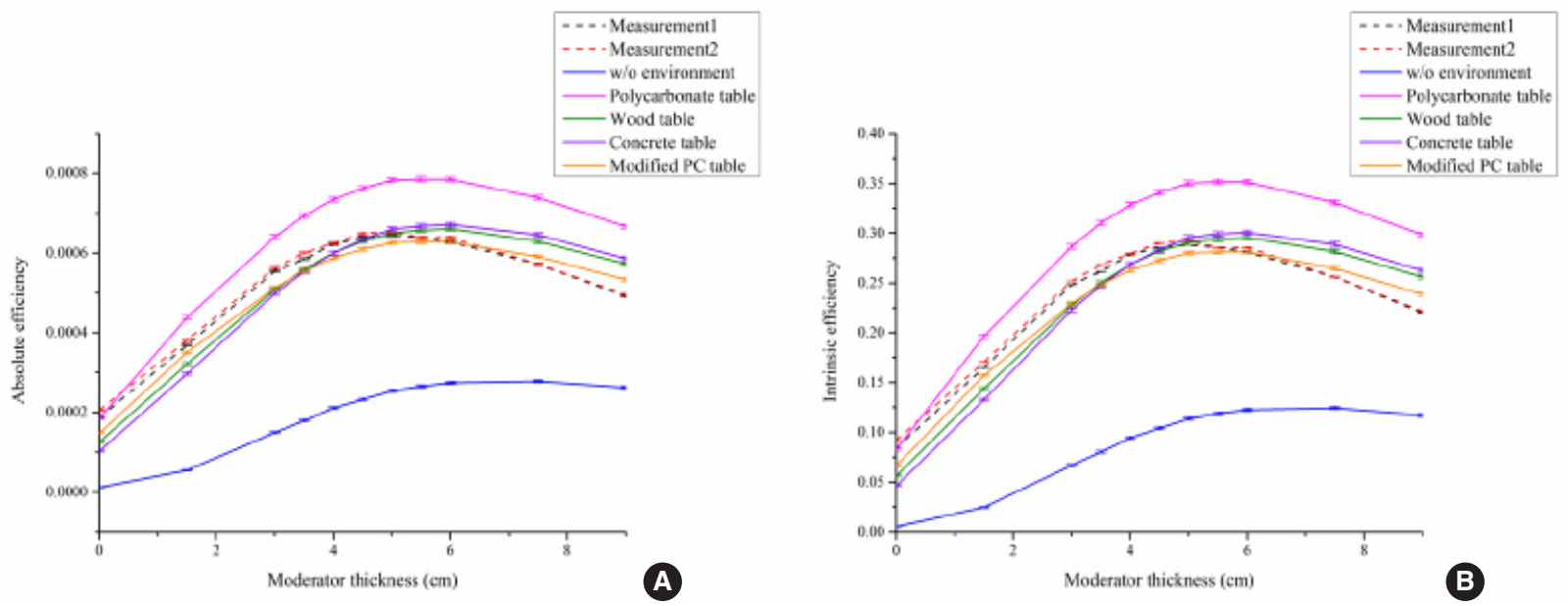

Fig. 10. (A) Absolute and (B) intrinsic neutron detection efficiency of CLYC at various moderator thicknesses; w/o environment means the table and the source holder were not considered in the simulation.

neutrons was estimated to be $7.5 \mathrm{~cm}$, based on the simulation without consideration of the environment, whereas the result from the experiment indicated that $\sim 5 \mathrm{~cm}$ gives the maximum counts of neutrons. Similar results were obtained in a previous study on the simulation of CLYC detector response to neutrons [12]. The previous study also showed a significant discrepancy between Monte Carlo simulation and measurements on ${ }^{252} \mathrm{Cf}$ source with PE moderators.

Thus, we attempted to model experimental environments to a reasonable degree. We included the source holder and table used for the measurement into the MCNP6 simulation. We assumed the source holder made of polycarbonate, and the table made of either wood, concrete or polycarbonate. As shown in Figure 9, just a simple implementation of experimental environments made substantial changes on the simulation result, and the simulation result made good estimations on neutron moderation trend. An optimal thickness of the PE moderator for neutron detection was estimated to be $\sim 5 \mathrm{~cm}$.
Table 3 shows simulation results on the absolute neutron detection efficiency with and without consideration of the experimental environment, compared with 2 sets of measurement results. The results are also illustrated in Figure 10. Error bars in Figure 10 represent 95\% confidence intervals given by the simulation and measurement counts. The absolute efficiency was calculated in a similar way as for gammas. Notably, the measurement results show quite different values from the MCNP simulations without environment, whereas simulation results with concrete and wood show less deviation from the measurement results. Simulation results with polycarbonate showed relatively higher values than measurement results, however, the overall shape of the curve for the normalized counts showed good agreements with measurement results in Figure 9. Thus, we multiplied a constant value, 0.8 on the polycarbonate results, represented in Figure 10 as "modified PC". The results showed good agreements with the measurement result, which reconfirm 
consistent $\sim 20 \%$ discrepancy as we observed in gamma-ray measurements and simulations.

We consider the actual workbench used in the experiment may be composed of materials similar to polycarbonate, however, a perfect replication of the structure and the composition of the experimental environment is practically impossible. Nevertheless, the simulation results show promise that the simulation can make good estimations on the overall trend of the measurement result, if experimental environments are properly modeled to a reasonable extent.

\section{Conclusion}

An initial study for the CLYC detector system was performed as a first implementation case of the detector system in Korea, to provide references for the future studies and applications. A commercial CLYC detector system obtained from BTI was characterized for its energy resolution and detection efficiency, and Monte Carlo simulations on the detector system were validated experimentally. Results showed good agreements in overall characteristics of the gamma and neutron detection efficiencies, with consistent $\sim 20 \%$ discrepancy. Furthermore, moderation of neutrons emitted from ${ }^{252} \mathrm{Cf}$ showed similarities between the simulation and the experiment, in terms of their relative ratios depending on the thickness of the PE moderator. Validation of the simulation results in overall trend of the CLYC detector behavior will provide the fundamental basis and validity of follow-up Monte Carlo simulation studies for the development of our dualparticle imager using a rotational modulation collimator [6-7].

\section{Acknowledgements}

This work was supported by the Nuclear Safety Research Program through the Korea Foundation of Nuclear Safety (KOFONS), granted financial resource from the Nuclear Safety and Security Commission (NSSC), Republic of Korea (No. 1403024).

\section{References}

1. Glodo J, Higgins WM, van Loef EVD, Shah KS. Scintillation properties of 1 inch $\mathrm{Cs}_{2} \mathrm{LiYCl}_{6}$ :Ce crystals. IEEE Trans. Nucl. Sci. 2008;55(3):1206-1209.

2. Glodo J, Hawrami R, van Loef E, Shirwadkar U, Shah KS. Pulse shape discrimination with selected elpasolite crystals. IEEE Trans. Nucl. Sci. 2012;59(5):2328-2333.

3. Glodo J, Hawrami R, Shah KS. Development of $\mathrm{Cs}_{2} \mathrm{LiYCl}_{6}$ scintillator. J. Cryst. Growth. 2013;379:73-78.

4. Smith MB, Achtzehn T, Andrews HR, Clifford ETH, Ing H, Kovaltchouk VD. Fast neutron spectroscopy using $\mathrm{Cs}_{2} \mathrm{LiYCl}_{6}$ :Ce (CLYC) scintillator. IEEE Trans. Nucl. Sci. 2013;60(2):855-859.

5. Pelowitz DB. MCNP6 ${ }^{\mathrm{TM}}$ user's manual version 1.0. LA-CP-13-00 643. Los Alamos National Laboratory. 2013;195-210.

6. Kim HS, Choi HY, Lee G, Ye SJ, Kim G. Monte Carlo simulation of rotational modulation collimator (RMC) patterns for the gamma-ray/neutron dual-particle imager. IEEE NSS/MIC/RTSD Conference. Proc. San Diego, CA. October 31-November 7, 2015.

7. Kim HS, Ye SJ, Shin Y, Lee G, Kim G. Radiation imaging with a rotational modulation collimator (RMC) coupled to a $\mathrm{Cs}_{2} \mathrm{LiYCl}_{6}: \mathrm{Ce}$ (CLYC) detector. J. Korean Phys. Soc. 2016;69(11):1644-1650.

8. Rizwan U, Chester A, Domingo T, Starosta K, Williams J, Voss P. A method for establishing absolute full-energy peak efficiency and its confidence interval for HPGe detectors. Nucl. Instrum. Methods A. 2015;802:102-112.

9. McDonald BS, Myjak MJ, Hensley WK, Smart JE. System modeling and design optimization for a next-generation unattended sensor. IEEE Trans. Nucl. Sci. 2013;60(2):1102-1106.

10. Won BH, Seo H, Lee SK, Park SH, Kim HD. Evaluation of neutron detection efficiency of the unified non-destructive assay using MCNPX code. J. Radiat. Prot. Res. 2013;38(4):172-178.

11. Lawrence Livermore National Laboratory. Simulation of neutron and gamma ray emission from fission and photofission. UCRL-AR-228518. 2014;1-29.

12. Bourne MM, Mussi C, Miller EC, Clarke SD, Pozzi SA, Gueorguiev A. Characterization of the CLYC detector for neutron and photon detection. Nucl. Instrum. Methods Phys. Res. Sect. A. 2014;736:124-127. 